\title{
Does Complex Learning Require Complex Connectivity?
}

\author{
Carlos Rubén de la Mora-Basáñez ${ }^{1,2}$, \\ Alejandro Guerra-Hernández ${ }^{1}$, and Luc Steels ${ }^{2}$ \\ ${ }^{1}$ Departamento de Inteligencia Artificial \\ Universidad Veracruzana \\ Facultad de Física e Inteligencia Artificial \\ Sebastián Camacho No. 5, Xalapa, Ver., México, 91000 \\ \{cdelamora, aguerra\}@uv.mx \\ 2 Artificial Intelligence Laboratory \\ Vrije Universiteit Brussel \\ Pleinlaan 2, 10G725, Brussels, Belgium 1070 \\ luc@arti.vub.ac.be
}

\begin{abstract}
Small World and Scale Free network properties characterize many real complex phenomena. We assume that low level connectivity with such topological properties, e.g., anatomical or functional connectivity in brains, is compulsory to achieve high level cognitive functionality, as language. The study of these network properties provides tools to approach different issues in behavior based Artificial Intelligence (AI) that usually have been ill defined, e.g., complexity and autonomy. In this paper, we propose a model in which situated agents evolve knowledge networks holding both Small World and Scale Free properties. Experimental results in the context of Pragmatic Games, elucidate some required conditions to obtain the expected network properties when performing complex learning.
\end{abstract}

\section{Introduction}

Behavior based AI 6[21|23] claims the necessity to work with autonomous, embodied and situated systems, always arguing biological referents. Similar approaches include development 281915] as an ineluctable issue. However, concepts such as embodiment 27, autonomy [7, or even the epigenetic [13|14, are ill defined or even are used with different connotations.

The validation of behavior based AI systems involves comparisons with the functionality of observed systems, being difficult to define general measures. Despite that, a kind of universal way of organization in connectivity has been recently found in a huge number of real complex phenomena: the complex networks 26/42|18 exhibiting Small-World and Scale-Free properties.

Complex networks are present at different levels of cognitive functionality, from functional connectivity in brains [10, to high level cognitive behavior as language 11. Although not yet well understood, this means that high level functionality is grounded on appropriate low level connectivity. 
In this paper, we present a model in which an artificial situated agent develops knowledge networks exhibiting complex topologies. Experimental results, using Pragmatic Games [8], support explanations about the required conditions to obtain networks with the expected properties, i.e., Small World and Scale Free, relating degree distribution and sensing; clustering coefficient and biological motivations; goals, acquired knowledge, and attentional focus. This constitutes a relevant advance in the understanding of how may low level connectivity emerge in artificial agents.

Organization is as follows: Section 2 introduces complex networks. Section 3 presents the methodology of Pragmatic Games. Section 4 discusses experimental results. Finally, section 5 concludes the paper.

\section{Complex Networks}

Many interesting phenomena involving networks with a huge number of nodes does not hold a random topology, and their degree distribution does not follow a normal distribution. Such networks hold a complex topology [26|4|2]18, where characteristic parameters are statistical macro variables describing the whole network and representing a fingerprint of complexity. Small World networks exhibit short characteristic path lengths but high clustering coefficients, when compared to random networks. These measures are used to determine how far of randomness, or near to order, a network is. Interesting networks were found in the middle between randomness and complete order. Scale Free networks exhibit power-law degree distributions, rooted in their growth mechanism 3 .

Phenomena holding the Small World and Scale Free properties include social, informational, technological, and biological networks, in fields as diverse as the WWW [1, metabolic networks [12, human language [11, scientific collaboration networks [5], brain networks in mammalians [25], or functional brain networks in humans 10. Clear examples of how high level cognitive functions emerge from low level connectivity are present in brain connectivity, particularly in human cerebral cortex [10, and also in other mammalians [25]; and in language networks [22].

The Scale Free property of brains explain the coexistence of functional segregation and integration, as well as redundancy and efficiency in information transmission. The power-law in degree distribution affects the functional impact of brain lesions: few highly connected nodes are vulnerable to damage, which explains de small impact of damage on random lesions. Cortical areas in mammalian brains exhibit attributes of complex networks. The distribution of functional connections and the probability of finding a link versus distance are both Scale Free. Additionally, the characteristic path length is small and the clustering coefficient is high, when compared with random graphs. Short path length captures potential functional proximity between regions. High clustering measures the degree of which a particular area is part of local collective dynamics. Frequent connectivity in all shortest paths linking areas, explains structural stability and efficient working of cortical networks. 
Human language is an important example of complex cognitive functionality with complex networks properties, mounted on complex connectivity (the brain itself). This connectivity allows the fast and robust construction of a huge variety of sentences from limited number of discrete units (words). Co-ocurrence, syntactic, and semantic networks shown to hold complex connectivity 22, having power-law degree distributions $(\gamma \sim 2.2-3.0)$, high clustering coefficient $\left(C / C_{\text {rand }} \sim 10^{3}\right)$, and small path-length. Hub deletion have the effect of loosing optimal navigation in co-occurrence networks; articulation loss in the case of syntactic networks; and conceptual loose plasticity in the case of semantic networks. Graphs of word interactions 11] show small-world properties (high clustering coefficient) and scale-free distributions $(\gamma \sim 1.5-2.0)$. Based on the significance profile of small sub-graphs from different languages, common local structure in word adjacency networks is also observed [17. This allows the definition of universal classes of networks by local motif statistics [16].

The study of complex networks is an active field which aims to understand complex phenomena, focusing in finding statistical properties to characterize the structure and behavior of such networks; creating models to explain such parameters; and predicting their behavior based on topological properties and local rules for nodes and links. In particular, we are interested in the necessary conditions that enables a situated agent to develop complex networks and how to obtain them.

\section{Pragmatic Games}

Pragmatic Games [8], inspired in language games [24, are used to explore how an artifical agent can develop complex knowldge networks. They offer a methodology by which situated agents are immersed in similar but not identical repetitive situations to perform complex spatial learning. With these games we are able to control, test, and make measurements of the network development. Three games (Fig. 1) are defined:

- Focusing game. One agent having only one eye and its visual field. The game starts setting an object within an environment in a random place. The eye moves randomly, if the fovea "sees" the object, the game is restarted.

- Grasping game. One agent having one eye and one hand. The game restarts when the hand reaches the object.

- Feeding game. One agent having one eye, one hand, and a mouth. When the hand passes over the object, the agent closes its hand and the object is attached to it. The random movements continue until the hand (with the object) reaches the mouth. At this moment, the game restarts.

The knowledge acquired by the agents while performing complex learning is represented as an evolving network of affective states, processed by a mechanism emulating epigenesis (see subsection 3.2). The morphology of the agent, the specific interaction within the environment (the game), together with the mechanism proposed to grow up the networks, establish the minimal conditions discriminating the games that produce complex networks and those that do not. 

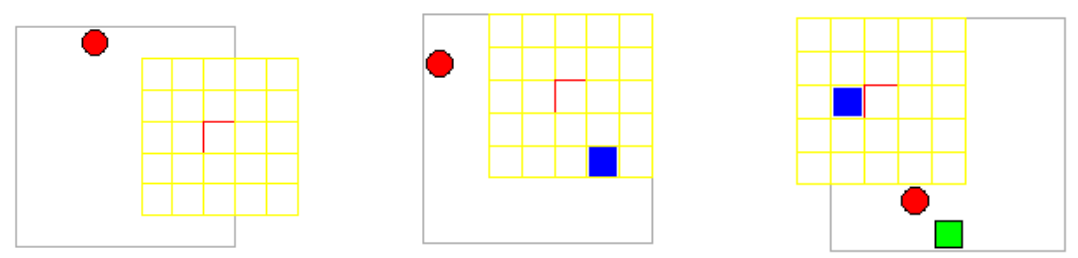

Fig. 1. Pragmatic Games, from left to right: Focusing (visual field), Grasping (visual field and hand) and Feeding (visual field, hand, and mouth). In all games the object is the circle.

\subsection{The Agent}

Our agent has 77 bit sensors: 75 bits for a $5 \times 5$ red $(\mathrm{R})$, green $(\mathrm{G})$, and blue $(\mathrm{B})$ sensitive visual field; one bit for a $1 \times 1$ blue "hand"; and one for a $1 \times 1$ green "mouth". The agent has four actuators: two for the eye and two for the hand. Each actuator has three possible states: do nothing, up/right, and down/left. The mouth is always fixed. An actuation is specified by a set of four values $\left(e_{x}, e_{y}, h_{x}, h_{y} \in\{1,0,-1\}\right)$. The agent moves randomly choosing one of the three possible states of each one of the actuators, considering these states as random variables with uniform distribution.

The agent has a set of distinguishable innate affective states [20], called biological motivations. These biological motivations are mapped to a 5-bit vector: Three bits for detecting RGB in the fovea; one for the hand holding an object, and other for the presence of the object in the mouth. Therefore, there are 32 possible biological motivations. These biological motivations do not have any appetitive or aversive character, they are only distinguishable. At the beginning they are not related with any sensorial state, the relation must be established by the mechanism which makes the network grow up.

\subsection{Network Growth Mechanism}

Every time the agent experiences a particular biological motivation, this motivation and the sensing state (77 bit string) is saved. Thus, every biological motivation has associated a record of sensing vectors. This process does not affect the network. Any biological motivation may give place to an affective state or a potential affective state, which is incorporated to the network by two mechanisms: 1) Detecting affective states from biological motivations. The affective state represents the set of sensing bits always present in the associated record, every time the biological motivation has been experienced, or; 2) Detecting potential affective states. If the sensing state at time $t$ corresponds to an affective state, then a node corresponding to the sensing state in the time $t-1$ is incorporated, as well as the directed arc between the nodes (representing the actuation).

A potential affective state could become affective state if its frequency exceeds some value. If the values are too small, noise can be learned. If the values are too 
big, then it takes more time to learn. This also happens for other parameters of the model.

The arcs joining nodes are incorporated in the network in two ways: 1) When a potential affective state is reached, as mentioned above, and; 2) When the agent experiences two consecutive affective or potential affective states.

Arcs are labeled as frequent or codified. Once an arc exists between two nodes, some statistics (frequency and actuation) are updated if it is traversed. Its label is then computed in the following way:

1. If the frequency of occurrence for an arc is higher than a given value, it is labeled as a frequent arc. The distribution of probabilities for the actuators is computed from the history and saved in the arc in the form: $\left\{p\left(e_{x}=-1\right)\right.$, $\left.p\left(e_{x}=0\right), p\left(e_{x}=1\right)\right\},\left\{p\left(e_{y}=-1\right), p\left(e_{y}=0\right), p\left(e_{y}=1\right)\right\},\left\{p\left(h_{x}=-1\right)\right.$, $\left.p\left(h_{x}=0\right), p\left(h_{x}=1\right)\right\},\left\{p\left(h_{y}=-1\right), p\left(h_{y}=0\right), p\left(h_{y}=1\right)\right\}$. The distributions are normalized.

2. If one of the three probabilities in each one of the four triplets of a frequent arc is higher than a threshold, all the triplet is replaced with one code, representing the associated winning movement for the correspondent actuator. For example, if the distribution of probabilities for the eye in the $x$ direction is $\left\{p\left(e_{x}=-1\right), p\left(e_{x}=0\right), p\left(e_{x}=1\right)\right\}=\{0.1,0.3,0.7\}$, then the triplet is replaced by a $1\left(p\left(e_{x}=1\right)=0.7\right.$ is the highest probability over the threshold).

\subsection{Attentional Focus}

Attentional focus is a behavior modifier [9] given as a number between $[0,1]$ representing the probability to undo the last movement performed. Thus, a 0.5 attentional focus means that the agent has a 0.5 of probability to revisit the last sensorial state. Each one of the pragmatic games was performed using different attentional focus values: $0.0,0.25,0.50$, and 0.75 . A 0.0 value is equivalent to random movement. A 1.0 value was not used, because it implies an infinite loop between two sensing states. These attentional focus values are fixed during all the experiments.

\section{Results}

Experiments showed that the networks evolved by the situated agents depend on the played game and the mechanism to make the network grow up. They also showed that wiring is made in terms of experiences. First, the complexity of interactions was found relevant to evolve complex networks, relating degree distribution and sensing. Second, biological motivations affect the clustering coefficient of the obtained networks. And third, attentional focus affects the number of goals achieved and the amount of knowledge acquired. 


\subsection{Degree Distribution and Sensing}

Power-law degree distribution is a fingerprint to distinguish complex networks. This property reflects the existence of few highly connected nodes (hubs), and a lot of them few connected. This allows tolerance to random fails, but vulnerability if the failure affects a hub.

A substantial difference exists between unimodal and multimodal games. In the unimodal game (focusing) the resulting network is by no means complex. But in multimodal games (grasping and feeding) a power-law emerges (Fig. 2). The only difference between grasping and focusing games, is the $1 \times 1$ moving hand around the world, absent in the focusing game. It is not only the complexity of the environment what matters, but the complexity of the interactions.
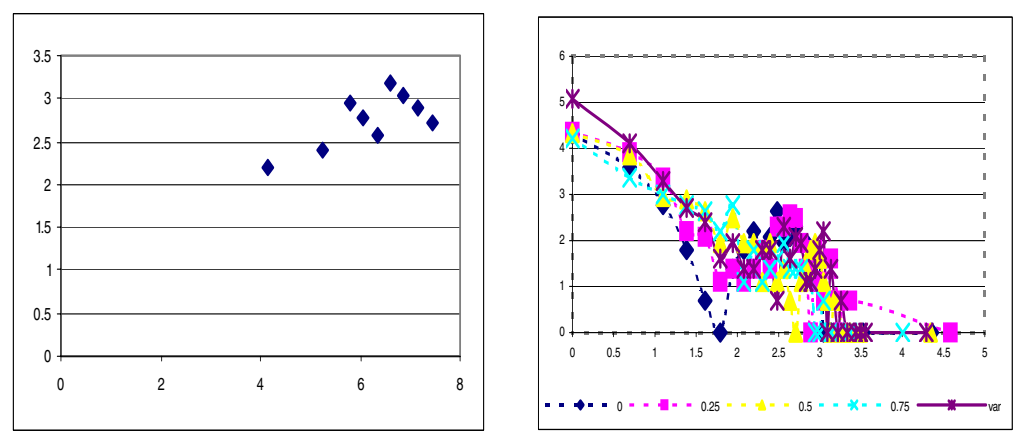

Fig. 2. Degree distribution for focusing game at left (degree $\times$ frec) and grasping game at right (ln degree $\times \ln f r e c)$. The unimodal-multimodal character of the game affects the complexity of the resulting network.

\subsection{Clustering Coefficient and Biological Motivations}

Clustering coefficient gives a measure of the possibility of being part of a local collective dynamics. In some way, it measures the integration of elements in a network. It is shown that clustering coefficient in the multimodal games is high when compared with a random graph (Fig. 3). It is also shown that this measure depends on the growing mechanism itself, particularly on the consideration of biological motivations (Fig. 4).

\subsection{Goals, Acquired Knowledge, and Attentional Focus}

The number of played games in each run can be understood as the number of goals achieved by the agents. The experiments show that the rate of growth of the number of goals in time is similar for all the games. A lower focus, as expected, is associated with a higher probability to advance, resulting in more games played (Fig. [5). 

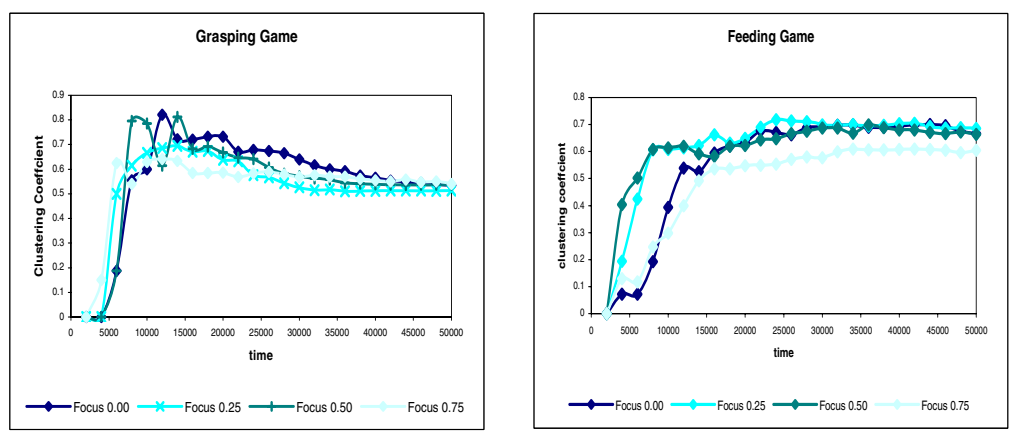

Fig. 3. Clustering coefficient in grasping game (left) and feeding game (right) is high when compared with an equivalent random graph

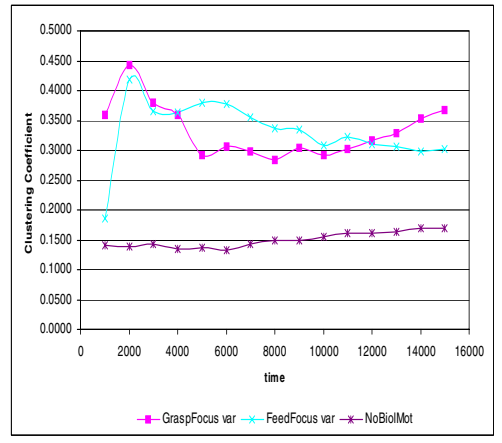

Fig. 4. Clustering coefficient and biological motivations. When biological motivations are not considered in the epigenetic mechanism to evolve the net, the clustering coefficient is very low.
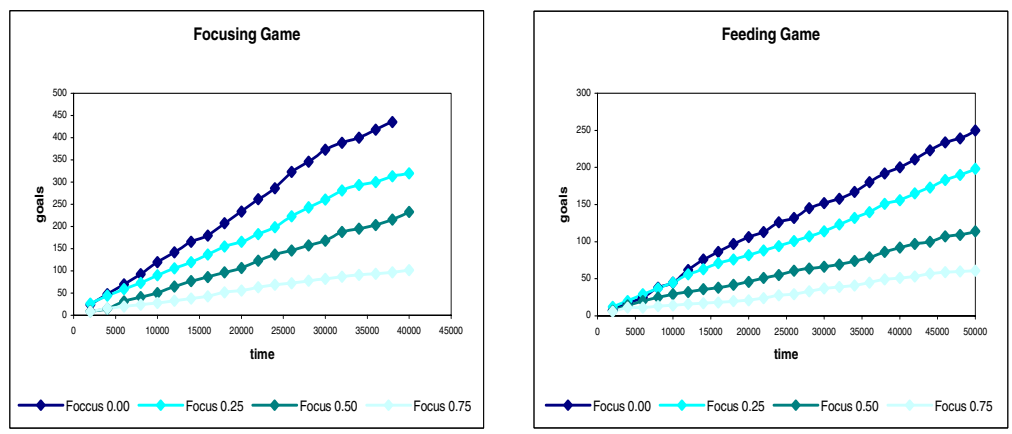

Fig. 5. The number of games played (goals) by the agent is similar when focus is changed, both for focusing and feeding games 
However, this tendency is different when the amount of knowledge acquired is considered. A fact is identified as two affective states connected by a coded arc. The number of facts is a measure of the quantity of acquired knowledge. It depends on the focus value, but its effect varies with the played game.

In our unimodal game, more goals and knowledge are attained by the agent for lower focus values. In the multimodal games the opposite is observed. This seems to be the source of the well known exploration/exploitation trade-off in learning agents. If the game is multimodal, the agent requires different behavior to achieve goals and to acquire knowledge. In the unimodal game, this situation does not exist (Fig. 6).
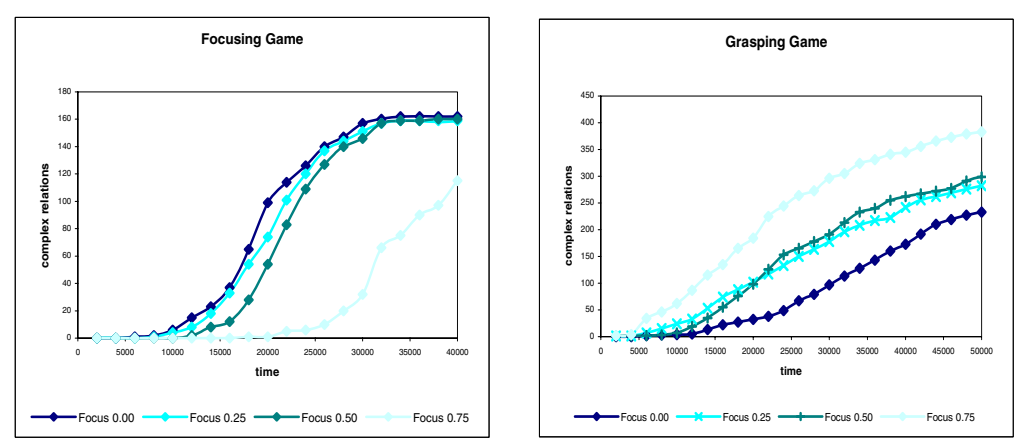

Fig. 6. The knowledge acquired depends both on the game played and the focus value

\section{Conclusions}

We use pragmatic games as a methodology to study knowledge development in situated agents. Knowledge is represented as an evolving network resulting from a mechanism emulating epigenesis, which is enacted by experiences. The knowledge acquired in this way is complex, because it relates spatial rules in appropriate way. The proposed mechanism to evolve knowledge includes innate biological motivations recognition, and the incorporation of nodes and links in steps. Network analysis and the associated measures are applied on the corpus of acquired knowledge.

In our experiments, clustering coefficient is large (a Small World property), compared with random graphs. This result is due to the proposed epigenetic mechanism. Particularly, because the innate biological motivations starting the process give rise to hubs in the network.

Knowledge networks in games involving multimodal agents exhibit powerlaw distribution (Scale Free property), whereas the games played by unimodal agents do not. This means that complex learning arises independently of the complexity of the environment, but depending on the interaction the agent has with it. Connectivity distribution in our unimodal game is finite and well defined, 
no matter the size of the eye. If the hand is introduced, the number of states increases significantly, emerging a complex network.

To observe how might the agent behavior affect acquired knowledge, we use a behavior modulator called attentional focus. If we consider the number of achieved games as the number of goals achieved by the agent, we observe that the focus value have the same impact for both unimodal and multimodal games, i.e., lower focus values result in more achieved games. However, the number of incorporated facts in the network depends on the modality of the game. For the unimodal game, lower focus values result in higher number of facts; but for the multimodal games, the inverse relation is observed. Thus, the modality of the game seems to be the source of the well known exploitation-exploration trade-off in learning agents.

The epigenetic mechanism used to make grow the network is suitable, in the sense that the topology of the generated networks evolves showing complex network properties. These results suggest that artificial agents must consider biological motivations in their learning mechanisms and be multimodal.

Complex connectivity in artificial agents seems to be necessary, because properties considered as essential in the definition of such agents, emerge in natural way from complex connectivity. For an artificial agent, complex topology represents the potentiality to exhibit complex functionality, i.e, the dynamics that becomes exploitation of the acquired knowledge.

Future work will consider cognitive autonomy which enables the agent to adapt its behavior to maximize knowledge acquisition, so that the amount of acquired knowledge becomes a behavior modulator. The effect of the relations between knowledge and behavior will be quantified and analyzed in terms of complex networks properties.

\section{References}

1. R. Albert, H. Jeong and A-L. Barabási, The diameter of the World Wide web, Nature, 401:130-131 (1999)

2. R. Albert and A-L. Barabási, Statistical Mechanics of Complex Networks, Reviews of Modern Physics, 74, 47 (2002)

3. A-L. Barabási and R. Albert, Emergence of scaling in random networks, Science, 286:509-512 (1999)

4. A-L. Barabási, Linked: The New Science of Networks, Perseus (2002)

5. A-L. Barabási et al., On the topology of the scientific collaboration networks, Physica A, 311:590-614 (2002)

6. R.A. Brooks, Cambrian Intelligence: The early history of the New AI, MIT Press (1999)

7. J. Collier, Autonomy and Process Closure as the basis for functionality, Annals of the New York Academy of Sciences, J.L.R. Chandler and G. Van der Vijver (eds.), 901:280-290, New York, USA (2000)

8. C. R. de la Mora-Basáñez, C. Gesherson, and V. A. García-Vega. Representation development and behavior modifiers. In C. Lemaître, C. A. Reyes, and J. A. González, editors, Adavances in Artificial Intelligence: Iberamia 2004, volume 3315 of LNAI, pages 504-513, Berlin, Germany, 2004. Iberamia, Springer Verlag. 
9. D. Dorner and K. Hille. Artificial souls: Motivated emotional robots. In IEEE Conference Proceedings, International Conference on Systems, Man, and Cybernetics; Intelligent Systems for the 21st Century volume 4, pages 3828-3832, Vancouver, Canada, 1995

10. V.M. Eguiluz et al., Scale-free brain functional networks, Phys. Rev. Letters, 92, $018102(2005)$

11. R. Ferrer-i-Cancho and R.V. Sole, The small world of human language, Proceedings of The Royal Society of London, Series B, Biological Sciences, 268(1482):2261-2265, UK (2001)

12. H. Jeong et al., The large-scale organization of metabolic networks, Nature, 407:651-654 (2000)

13. J. Lederberg, The Meaning of Epigenetics, The Scientist 15(18):6 (2001)

14. J.M. Levenson and D. Sweatt, Epigenetic mechanisms in memory formation, $N a$ ture 6:109-119, February (2005)

15. A. Mcintyre, F. Kaplan and L. Steels, Crucial Factors in the Origins of WordMeaning, The Transition to Language, A. Wray (ed.), pages 252-271, Oxford University Press, Oxford, UK (2002)

16. R. Milo et al., Network Motifs: Simple Building Blocks of Complex Networks, Science 298:824-827, October (2002)

17. R. Milo et al., Superfamilies of evolved and Designed Networks, Science 303: 1538-1542, Mach (2004)

18. M.E.J. Newman, The structure and function of complex networks, SIAM Review, 45(2):167- 256 (2003)

19. C.G. Prince, Introduction: The Second International Workshop on Epigenetic Robotics, Lund University Cognitive Studies 94, Lund, Sweden (2002)

20. M. Scheutz, The Evolution of Simple Affective States in Multi-Agent Environments, Proceedings of AAAI Fall Symposium 01, AAAI Press, USA (2001)

21. T. Smithers, Taking Eliminative Materialism Seriously: A Methodology for Autonomous Systems Research, Toward a Practice of Autonomous Systems: Proceedings of the First European Conference on Artificial Life, F.J. Varela and P. Bourgine (eds.), MIT Press / Bradford Book, pages 31-40 (1992)

22. R.V. Sole et al., Language Networks: their structure, function and evolution, Santa Fe Institute Working Paper 05-12-042, Santa Fe, CA., USA (2006)

23. L. Steels and R. Brooks, The Artificial Life route to Artificial Intelligence: Building situated embodied agents, Lawrence Erlbaum Ass. (1995)

24. L. Steels, Self-organizing vocabularies, Proceedings of Alife V, C. Langton and T. Shimohara (eds), pages 179-184, Nara Japan (1996)

25. O. Sporns et al.,Organization, development and function of complex brain networks, Trends in Cognitive Sciences, 8:418-425 (2004)

26. D. Watts, Small-Worlds. The dynamics of networks between order and randomness, Princeton Studies in Complexity, Princeton University Press, USA (1999)

27. T. Ziemke, Are Robots Embodied?, Proceedings of the First International Workshop on Epigenetic Robotics: Modeling Cognitive Development in Robotic Systems, Lund University Cognitive Studies 85, Lund, Sweden (2001)

28. J. Zlatev and C. Balkenius, Introduction: Why 'epigenteic robotics'?, Proceedings of the First International Workshop on Epigenetic Robotics: Modeling Cognitive Development in Robotic Systems, Lund University Cognitive Studies, 85:1-4, Lund, Sweden (2001) 